\title{
ANÁLISE DO AMBIENTE COSTEIRO E MARINHO, A PARTIR DE PRODUTOS DE SENSORIAMENTO REMOTO NA REGIÃO DE SÃO BENTO DO NORTE, NE BRASIL
}

\author{
Werner Farkatt Tabosa ${ }^{1}$, Venerando Eustáquio Amaro² e Helenice Vital ${ }^{1,2}$ \\ Recebido em 6 maio, 2005 / Aceito em 20 abril, 2006 \\ Received on May 6, 2005 / Accepted on April 20, 2006
}

ABSTRACT. This work characterizes the coastal and marine environment morphology of the São Bento do Norte area (NE Brazil) based on remote sensing analysis. Applying digital image processing techniques, that were georeferenced, rectified and processed. The integration of field data to the resulting images (RGB-5-2-1, HSI-PC6-4-2, PC2 e PC4) allowed the identification of the main submerged features and detection of environmental changes on the coastal zone. The area is located in Potiguar Basin between a complex system of faults and grabens/horsts. It is assumed that this fault system has had influence on of the generation morphology and control several bottom features. The recognition of submerged seabottom features as dunes, beachrocks and reefs, was only possible due to the high transparency of the water in the area that makes possible the mapping of the bottom morphology up to $25 \mathrm{~m}$ depth. Thus, the orbital images have been used with success in the mapping of the inner shelf of Northeast.

Keywords: remote sensing, coastline, continental shelf, morphology, Northeastern Brazilian.

RESUMO. Neste trabalho é explorado de modo rápido e eficaz as características morfológicas do ambiente costeiro e marinho na região de São Bento do Norte/RN a partir de sensores remotos (SR). Aplicando técnicas do processamento digital de imagem (PDI), estas foram georreferenciadas, retificadas e gerados os padrões estatísticos. 0 produto resultante deste processamento (RGB-5-2-1, HSI-PC6-4-2, PC2 e PC4), integrado a informações de campo, resultou na identificação das principais feições submersas detecção de mudanças geoambientais desta costa. A área está inserida nos domínios da Bacia Potiguar, um complexo sistema de falhas e grabens/horsts. Pressupõe-se que este sistema de falhas tenha influenciado na geração e no controle de diversas feições de fundo. 0 reconhecimento de feições submersas, tais como dunas longitudinais e transversais, arenitos de praia e recifes, só foi possível devido à transparência da água na área facilitando o mapeamento da morfologia de fundo até a profundidade de $25 \mathrm{~m}$. Desta forma, o sensoriamento remoto tem sido utilizado com sucesso no mapeamento da plataforma interna do Nordeste Brasileiro.

Palavras-chave: sensoriamento remoto, linha de costa, plataforma continental, morfologia, Nordeste do Brasil.

Universidade Federal do Rio Grande do Norte - UFRN, Cx. Postal 1639, 59.072-970, Natal, RN, Brasil. Telefone: ++55 (84) 215-3727 R-13, 215-3808 R-213; Fax: +55 (84) 215-3683 R-11-E-mails: farkatt@yahoo.com / amaro@geologia.ufrn.br / helenice@geologia.ufrn.br

1 Programa de Pós-graduação em Geodinâmica e Geofísica - PPGG.

${ }^{2}$ Departamento de Geologia/UFRN. 


\section{INTRODUÇÃO}

A região de São Bento do Norte e Caiçara do Norte está situada no litoral setentrional do Estado do Rio Grande do Norte, há uma distância de 170 km da capital Natal (Fig. 1), inserida em uma linha de costa submetida à ação contínua dos ventos alísios provenientes predominantemente de nordeste. Para meIhor elucidar a evolução paleogeográfica do Quaternário, fez-se necessário compreender a complexidade dos parâmetros hidrodinâmicos e a energia costeira atuantes nesta costa. Geralmente, esses fenômenos estão impressos no litoral sob forma de erosão ou deposição (Short, 1999; Tabosa et al., 2001a, 2002). Acreditase que as principais modificações ocorridas ao longo do litoral setentrional do Rio Grande do Norte foram influenciadas diretamente por processos oceânicos - intensidade e direção de correntes, ondas e marés, associados ou não a condicionantes meteorológicos - incidência pluviométrica, velocidade e direção dos ventos e/ou geológicos - elementos tectônicos regionais e locais (Tabosa et al., 2001a, b; Vital et al., 2003; Vital et al., 2006). Os efeitos destrutivos e/ou construtivos destes processos podem ser identificados ao longo de toda costa setentrional do Estado. Estudos objetivando entender as causas desta erosão são recentes (Tabosa et al., 2002; Vital et al., 2003; Vital, 2005) e mostram a necessidade de se conhecer melhor a plataforma interna adjacente.

Os estudos desenvolvidos na plataforma continental interna do Rio Grande do Norte se concentram em sua maior parte na região de Touros (leste de São Bento do Norte), Foz do Rio Açu e Galinhos-Guamaré (a oeste de São Bento do Norte). Neste contexto, destaca-se Vianna et al. (1991), que utilizando imagens de satélite Landsat 5-TM, identificaram antigas faces de praia e um campo de dunas transversais à $20 \mathrm{~m}$ de profundidade na plataforma continental ao largo da cidade de Touros-RN. Ainda utilizando imagens Landsat 5-TM, Solewicz (1989) evidenciou na plataforma norte um conjunto de feições submersas: paleocanais dos rios Mossoró e Açu; lineamento leste/oeste ao largo de Morrinhos e bancos arenosos submersos aproximadamente paralelos à linha de costa. Testa \& Bosence $(1998,1999)$ estudando a plataforma continental ao largo de Touros-RN, utilizando imagens Landsat 5-TM e amostragem, definiram diferentes zonas sedimentológicas, que ocorrem paralelas à linha de costa atual. Costa Neto (1997), com base em levantamentos batimétricos e amostragem pontual identificaram na plataforma adjacente à foz do rio Açu 13 fácies sedimentares, constituídos principalmente por sedimentos terrígenos e bioclásticos. Schwarzer et al. (2006), utilizam sísmica de alta resolução para estudos de evolução costeira na região da foz do rio Açu, identificando vales incisos e unidades sismoestratigráficas atribuídas à regressão pleistocênica e à transgressão holocênica. Röber (2001) mapeou na plataforma de Galinhos, campos de cordões arenosos, com extensão entre 12 e 20 km, com 300 a 500 m de largura, separada um do outro por uma distância aproximadamente de 1 a 1,5 km. Lima et al. (2001) e Lima (2004), em trabalhos realizados na plataforma adjacente a Galinhos e com base em imagem de satélite descrevem que os cordões identificados por Röber (2001), na realidade são uma série de estruturas sigmóidais interligadas. Alves et al. (2003) elaboraram uma carta temporal e uma análise geoambiental da dinâmica da foz do rio Piranhas-Açu com base em imagens Landsat 5-TM.

Tentando complementar e correlacionar as informações já existentes, este trabalho objetiva empregar outro conjunto de imagens (Landsat 7-ETM+) e composições e tratamentos semelhantes aos adotados para as imagens Landsat 5-TM. Esta opção foi adotada com 0 intuito de enfatizar a caracterização da plataforma interna e em alguns casos extrapoladas (separadamente) para zona costeira, de modo a determinar à dinâmica marinha e sua influência na zona litorânea, e assim subsidiar a elaboração de planos de gestão ambiental mais adequados à região.

\section{CONTEXTO GEOLÓGICO}

A área de estudo está inserida no domínio geológico da Bacia Potiguar (Fig. 2). A Bacia Potiguar situa-se no extremo leste da Margem Continental Brasileira, compreendendo uma parte emersa e outra submersa; seus limites ocupam grande parte do Estado do Rio Grande do Norte e uma porção do Estado do Ceará (Souza, 1982; Matos, 1987, 1992). Esta bacia apresenta uma complexa evolução tectônica (Matos, 1987; Fonseca, 1996), mesclando elementos tanto das zonas tectônicas equatoriais, quanto das zonas tectônicas do Atlântico sul, e constituindose uma das regiões sismicamente mais ativas do Brasil (Matos, 1987, 1992; Assumpção, 1992; Cremoni, 1995; Oliveira et al., 1996; Ferreira et al., 1998). Atualmente, é a segunda região produtora de petróleo do país, atrás somente da Bacia de Campos, com produção na bacia de 80 mil boe/dia e 3 milhões de $\mathrm{m}^{3}$ de gás, até o período de maio de 2003 (Soares et al., 2003).

A Bacia Potiguar é a mais oriental das bacias da Margem Equatorial brasileira, estando, geneticamente relacionada a uma série de bacias neocomianas intracontinentais que compõem sistema de riftes do NE brasileiro (Matos, 1987, 1992). Este sistema, análogo ao atual rift-valley do leste africano, compreende as Bacias do Recôncavo, Tucano, Jatobá, Araripe, Rio do Peixe. 


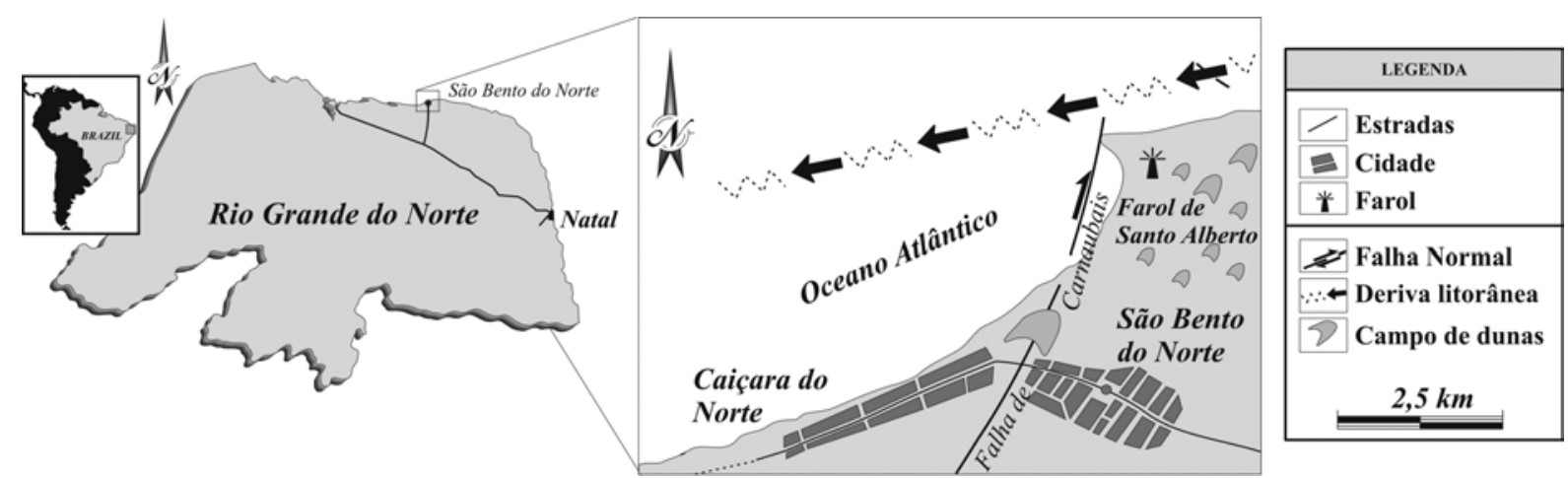

Figura 1 - Mapa de localização geográfica da área.

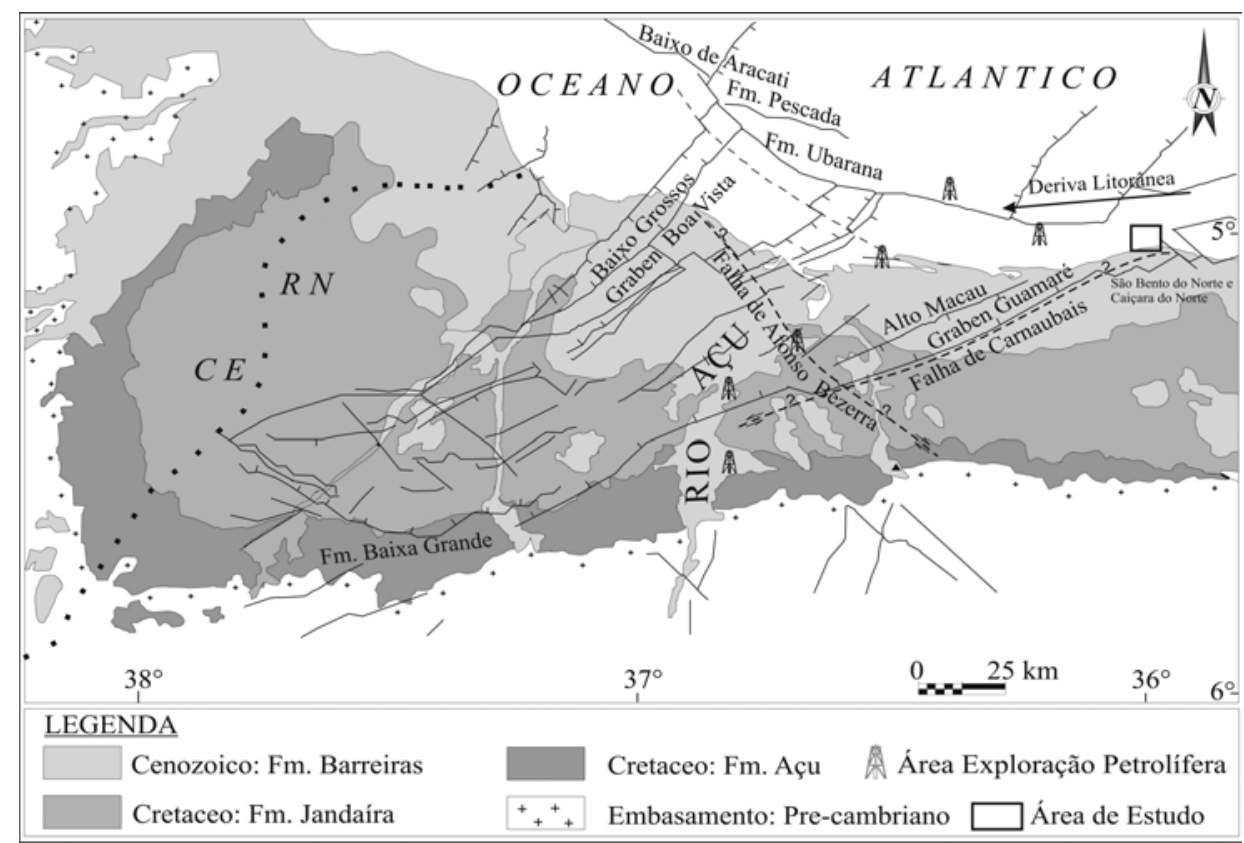

Figura 2 - Compartimentação tectono-estrutural do litoral norte, entre a Ponta do Mel e a Ponta dos Três Irmãos como consequiência do par conjugado definido pelas Falhas de Carnaubais e Afonso Bezerra no contexto da Bacia Potiguar (modificado de Bertani et al., 1990; Fonseca, 1996).

Entretanto, a Bacia Potiguar apresenta particularidades bem definidas, marcadas por uma tectônica tipo pull-apart para sua porção submersa, enquanto, na emersa, tem-se evidenciado um sistema de rifte tipo intracontinental (Bertani et al., 1990; Araripe \& Feijó, 1994).

As rochas sedimentares presentes nesta Bacia estão associadas a três seqüências deposicionais principais: uma continental, uma transicional e outra marinha. A seqüência continental é resultante da fase rifte de idade Neocomiano a Barremiano (Cretáceo inferior); a seqüência transicional de idade Aptiano/Eoptiano (Cretáceo médio), e a seqüência drifte de idade Albiano a Maastrichtiano/Campaniano e um sistema re- gressivo marinho, de idade Maastrichtiano/Campaniano a Plioceno/Paleoceno/Terciário (Souza, 1982; Bertani et al., 1990).

Araripe \& Feijó (1994) organizaram os litotipos da Bacia Potiguar desde 0 Neocomiano até 0 Terciário segundo três unidades principais, ordenadas da base para 0 topo, como: Grupo Areia Branca, Apodi e Agulha.

A geologia da área estudada está condicionada a dois grandes domínios, a plataforma continental e 0 ambiente costeiro. Tabosa et al. (2001b) descreve para a região de São Bento do Norte e Caiçara do Norte, nove unidades geológicas que são representados por aluviões, banco de arenitos, concheiros, depósitos de planície de maré, depósitos de praia, dunas móveis, paleocordões 
arenosos, paleodunas e paleodunas descaracterizadas (Fig. 3).

De acordo com as descrições de Tabosa (2000), a plataforma marinha adjacente à região de São Bento do Norte, encontrase formada por quatro domínios composicionais que compõem os sedimentos de fundo, distribuídos segundo os fácies: areias litoclásticas, areias litobioclásticas, cascalho e areias biolitoclásticos e sedimentos do Grupo Barreiras.

Depósitos holocênicos são constituídos por arenitos de praia (Fig. 3), de composição essencialmente quartoza, pobremente selecionados, de granulometria grossa a fina, com cimentação carbonática e estes estão distribuídos ao longo de toda a linha de costa. Os arenitos de praia podem ser observados tanto nos terraços antigos, como também, formando terraços aflorantes nas bordas dos canais, ou sotopostos às areias holocênicas. Apresentam um arcabouço composto por grãos tamanho areia de granulometria grossa e matriz fina, podendo apresentar também fragmentos de conchas com granulometria $>2,00 \mathrm{~mm}$ e cimentação carbonática (Bezerra et al., 1998; Stattegger et al., 2006; Caldas et al., 2006).

\section{MATERIAIS E MÉTODOS}

Neste trabalho foram empregadas imagens multiespectrais (bandas 1 a 5 e 7, do espectro visível-infravermelho próximo) do sistema Landsat 7-ETM+ (Órbita 215/064 de 12/Junho/2005, com hora de passagem aproximada às 10 h00 min \pm 15 min). No dia do imageamento, o instante de maré baixa, com altura aproximada de 0,6 m, obtido com marégrafo fundeado na cidade de Macau/RN, foi às 07h41min. 0 processamento digital das imagens multiespectrais foi realizado no software ER-Mapper v. 6.4, a integração e gerenciamento dos dados no software ArcView versão 3.2.

0 processamento de imagens envolveu as seguintes etapas:

- Pré-processamento das imagens: georreferenciamento das imagens digitais do Landsat 7-ETM+, que apresentaram a raiz da soma dos quadrados dos erros em relação à média (RMS) da ordem de 0,5 pixel, que permite a comparação/integração entre as imagens e a análise com outras camadas de dados;

- Cálculo estatístico das imagens: Empregada para se determinar à extensão de correlação entre as diferentes bandas espectrais, de tal forma que se possa determinar os diferentes atributos de correlação das imagens, com intuito de validar a análise por principais componentes (Tab. 1);

- Processamento digital de imagens: Composições colori- das no sistema de cores aditivas RGB, referente às cores aditivas Vermelho (Red) - Verde (Green) - Azul (Blue); composições coloridas no espaço IHS, relativo às propriedades das cores Intensidade (Intensity) - Matiz (Hue) Saturação (Saturation); Método de Razão de Bandas; e Análise por Principais Componentes.

\section{RESULTADOS E DISCUSSÕES}

A interpretação de algumas composições coloridas do sistema Landsat 7-ETM+ (de 12/Junho/2005) das bandas do visívelinfravermelho próximo destacou as informações relevantes sobre as unidades geoambientais presentes na área. Assim, foi possível a detecção das principais feições de fundo (dunas, linhas de arenitos de praia, recifes, etc.) impressas na plataforma continental. Na caracterização de tais feições fez-se necessário uma combinação de dados bibliográficos e de campo onde se avaliou os aspectos geomorfológicos, estruturais, ambientais e produtos resultantes de perfis sonográficos e batimétricos. A combinação dessas informações serviu de base para a interpretação da morfologia do fundo marinho e da zona costeira de São Bento do Norte e Caiçara do Norte.

Nas composições RGB-5-2-1 (Fig. 4), HSI-PC6-4-2 (Fig. 5), PC2 (Fig. 6) e PC4 (Fig. 7), foram delineadas diversas feições importantes em toda porção submersa, destacando-se: a pluma de sedimentos, diferentes tipos e tamanhos de dunas e linhas de arenitos de praia submersos.

A composição RGB-5-2-1 (Fig. 4) favoreceu a identificação das unidades geoambientais da porção costeira, baseado nos aspectos geomorfológicos, na cobertura vegetal e ocupação do solo (manguezais, solos expostos, culturas, etc.). De acordo com Alves et al. (2003) para áreas costeiras destaca-se 0 aumento no conteúdo na umidade do solo que gera uma diminuiç̧ão geral da reflectância em todos os comprimentos de onda. Ainda segundo estes autores, os compostos orgânicos dos solos (glucídeos, peptídeos, lipídeos, resinas, ácidos fúlvicos e húmicos, etc.) tendem a absorver na faixa do visível. A presença desses constituintes está diretamente relacionada à cor dos solos, descrita pela Carta de Munsell (Taylor, 1982). A imagem resultante proporcionou um ótimo resultado na detecção do limite terra/mar, onde as áreas emersas arenosas aparecem em cor branca (ponto 1) devido à alta reflectância dos sedimentos areno-quatzosos no visível-infravermelho próximo, além de sua pequena capacidade de retenção de água nos interstícios superiores (ponto 2). A composição colorida em RGB-5-2-1 proporcionou ainda, a identificação de alguns agrupamentos de vegetação, mostrando 


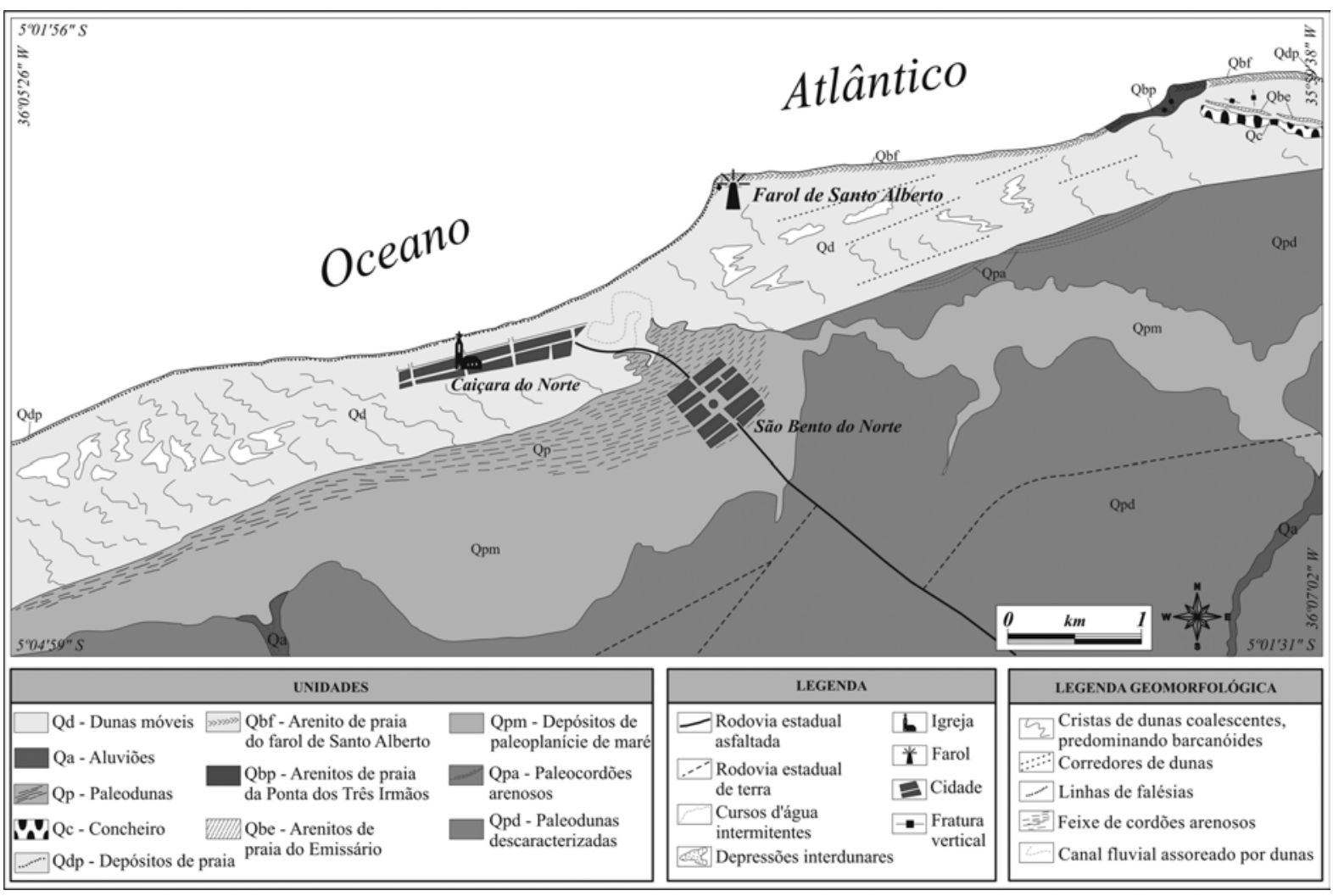

Figura 3 - Mapa geológico simplificado da região de São Bento do Norte/Caiçara do Norte, confeccionado a partir da interpretação de fotografias aéreas na escala de 1:40.000 (Cruzeiro do Sul, 1965; compilado de Tabosa, 2000).

Tabela 1 - Estatística da Cena 215/64 de 12/06/00 quanto aos valores dos autovetores das Principais Componentes. $\mathrm{PC}=$ Principal Componente

\begin{tabular}{|c|c|c|c|c|c|c|}
\hline & PC 1 & PC 2 & PC 3 & PC 4 & PC 5 & PC 6 \\
\hline Banda1 & 0.319 & -0.667 & 0.232 & -0.624 & -0.073 & -0.063 \\
\hline Banda 2 & 0.433 & -0.287 & 0.240 & 0.530 & 0.256 & 0.570 \\
\hline Banda 3 & 0.455 & -0.129 & -0.159 & 0.368 & 0.174 & -0.765 \\
\hline Banda 4 & 0.377 & 0.497 & 0.663 & -0.034 & -0.399 & -0.104 \\
\hline Banda 5 & 0.401 & 0.454 & -0.196 & -0.438 & 0.621 & 0.134 \\
\hline Banda 7 & 0.447 & 0.060 & -0.621 & -0.021 & -0.595 & 0.239 \\
\hline
\end{tabular}

um melhor resultado na discriminação da vegetação de dunas (ponto 2) e de áreas cultivadas (ponto 3). Na faixa costeira se destaca um conjunto de pequenas lagunas (ponto 4), de orientação geral de NE para SW, mantendo comunicação restrita com o mar, onde se observa uma variação em tons de azul claro a verde decorrente da reflectância na banda $2(0,52-0,60 \mu \mathrm{m})$ e proporcionado pela pouca profundidade da lâmina d'água (Fig. 4). Entretanto, estas feições se repetem tanto paralela à costa como dentro do continente na região do campo de dunas (lagoas interdunares; ponto 5). A área de dunas (Figs. 3 e 4) é marcada por uma extensa faixa de contornos irregulares evidenciada por tonalidades esbranquiçadas (Fig. 4; pontos 1 e 2).

Nas imagens HSI-PC6-4-2 (Fig. 5), PC2 (Fig. 6) e PC4 (Fig. 7), destacaram-se principalmente as feições contidas na zona marinha. Aplicando-se as técnicas do sensoriamento remoto (SR) e do processamento digital de imagens (PDI), foi possível destacar diversas feições submersas que se encontravam mascaradas pela lâmina de água e forte turbidez. Com a composição 


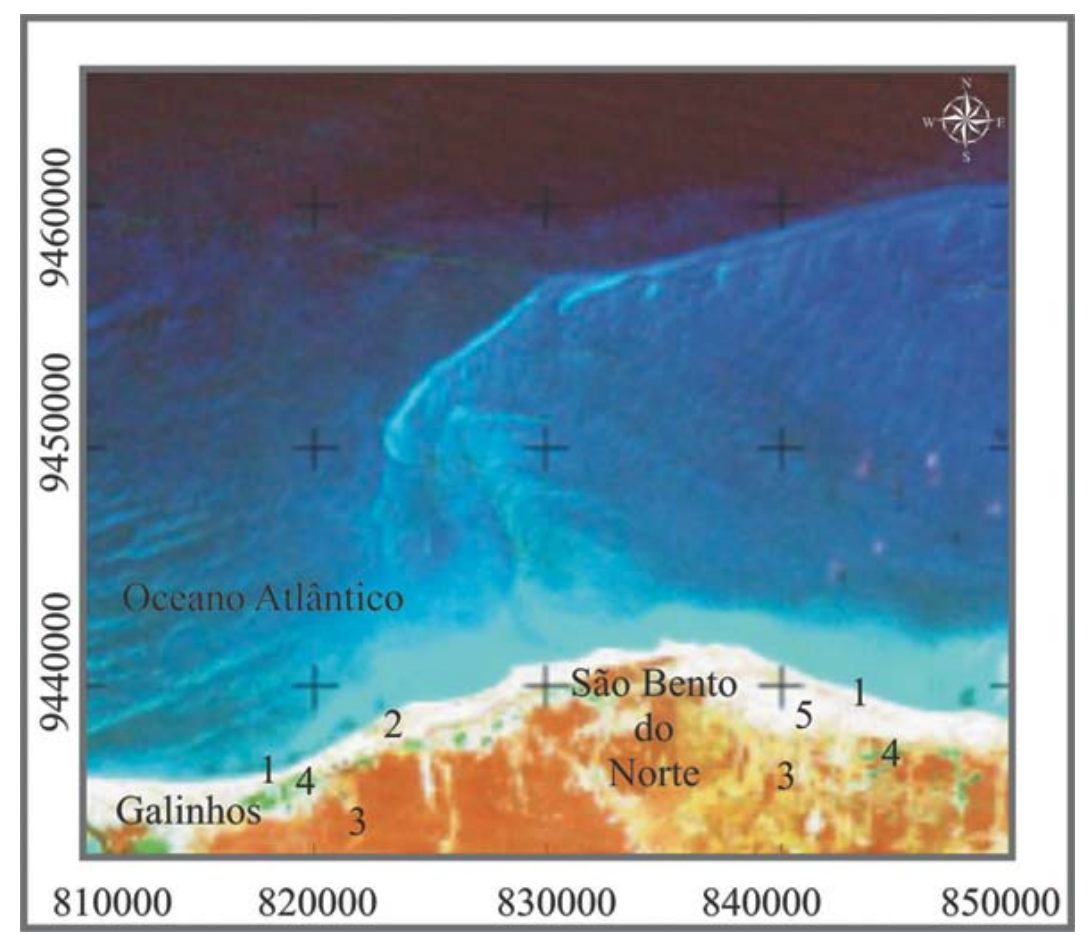

Figura 4 - Composição colorida RGB-5-2-1 da cena Landsat 7-ETM+ (WRS 215/064, de 12 de Junho de 2000, baixamar 7:41Hrs/AM. Passagem do satélite aproximadamente às 10:00Hrs/AM $\pm 15 \mathrm{~min}$ ) utilizada para caracterização da morfologia costeira. 1 - campo de dunas, 2 - campo de dunas vegetada, associada a corpos d'água, 3 -áreas cultivadas, 4 - lagunas, 5 - lagoas interdunas.

HSI-PC6-4-2 (Fig. 5, coordenada U.T.M. 9445000 N / 830000 E, da zona 24M), foi observando à presença de uma extensa pluma de sedimentos que bordeja toda a linha de costa. Esta pluma é resultado da remobilização dos sedimentos produzida por ação dos fortes ventos existentes na região e por ação das correntes costeiras que atuam de este para oeste (Tabosa \& Vital, 2004). Esta zona túrbida destaca-se na imagem por uma diversidade de cores que vai do branco ao vermelho-neón. Esta feição está realçada como uma extensa faixa (com cerca de $2 \mathrm{~km}$ de largura) contínua e paralela à linha de costa atual. Em outros pontos da imagem (Fig. 5) feições semelhantes também são identificadas numa curta faixa nas proximidades da coordenada U.T.M. 9450000 N / 830000 E, da zona 24M, estendendo-se até à cota batimétrica de 5 metros. A dispersão desta pluma ocorre nitidamente nas zonas mais profundas (6 a 8 metros). A variação de cores nesta faixa mais profunda é bastante diversificada, variando de azul turquesa ao azul anil, ao vermelho, ao magenta e ao branco. A dificuldade de identificação dos diversos elementos de paisagem e a diminuiç̧ão do nível de reflectância nesta porção mais profunda torna-se mais difícil em função da baixa penetração, das propriedades óticas dos objetos, da característica das substâncias dissolvidas e das características dos sedimentos em suspensão (Guyot, 1989).

Na porção W da imagem (Fig. 5) são observadas inúmeras dunas com direção N-NE, formando um extenso campo com aproximadamente $30 \mathrm{~km}$ de extensão por $16 \mathrm{~km}$ largura. Este campo cobre praticamente quase toda a plataforma interna (coordenada U.T.M. 9450000/9440000 N e 790000/820000 E da zona 24M). Entretanto, quando analisadas individualmente constata-se um conjunto de dunas segmentadas de pequeno a médio porte, com cristas sinuosas e flancos assimétricos. Na porção $\mathrm{E}$ da imagem outras dunas são identificadas, neste caso, o padrão de orientação sofre uma variação, gerando duas direções preferenciais: uma aproximadamente $\mathrm{E}-\mathrm{W}$ e outra $\mathrm{N}-\mathrm{S}$. Com auxílio de perfis sonográficos foi possível mapear a forma predominante dos flancos das dunas, que em geral são assimétricos e cristas sinuosas. As duas direções de dunas observadas nas imagens (HSI-PC6-42, Fig. 5; PC2, Fig. 6 e PC4, Fig. 7) provavelmente correspondem ao reflexo das duas correntes marítimas: uma costeira de maior intensidade ( $\mathrm{E}-\mathrm{W}$ ) e outra decorrente do ciclo de maré (N-S) de menor intensidade (Fig. 5).

Uma grande feição marinha raso destaca-se na porção central 


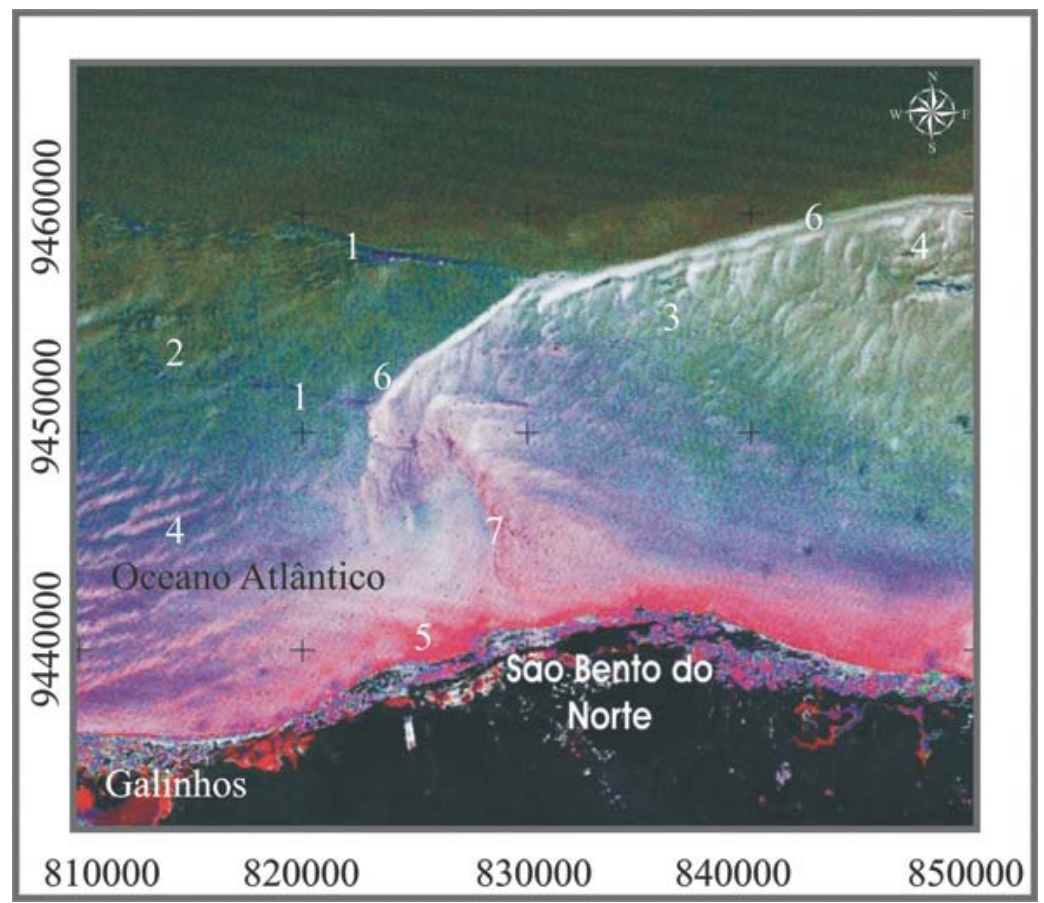

Figura 5 - Composição colorida HSI-PC6-4-2 do Landsat 7-ETM+ (WRS 215/064, adquirida em 12 de junho de 2000, baixamar 7:41Hrs/AM, passagem do satélite aproximadamente 10:00Hrs/AM $\pm 15 \mathrm{~min}$ ) destacando as seguintes feições. 1 - linha de arenitos de praia, 2 - estrutura recifal circular, 3 - dunas com cristas sinuosas e flancos assimétricos orientados preferencialmente N/NE, 4 - dunas com cristas sinuosas e flancos assimétricos orientados preferencialmente E/W e N/S, 5 - plumas de sedimentos em suspensão próximas à linha de costa, 6 - corpo arenoso 7 - paleocanal/zona de baixa profundidade.

da imagem (Figs. 4 a 7). Este feição estende-se desde 0 extremo $\mathrm{E}$ da imagem e assume uma feição curvada na zona central da imagem. De acordo com os estudos batimétricos e sedimentológicos, trata-se de um corpo arenoso, com cerca de $400 \mathrm{~m}$ de largura, tendo seus flancos marcados por uma topografia mais suave na porção interna (S) da feição e uma topografia mais acentuada com caimento abrupto na porção externa (N) em direção a quebra da plataforma. Corpos deste tipo já foram descritos na literatura (e.g. Dalrymple \& Rhodes, 1995; Snedden \& Dalrymple, 1999). Estas feições foram igualmente observadas nas imagens HSI-PC6-4-2 (Fig. 5), PC2 (Fig. 6) e PC4 (Fig. 7).

Linhas de arenitos de praia foram mapeadas ao longo de praticamente toda a costa Potiguar. Normalmente encontram-se parcialmente fragmentadas e interrompidas por extensas faixas de sedimentos recentes. Estes arenitos de praia (recifes) podem ser identificados nas imagens (Fig. 5), como extensos corpos alongados, bordejando a costa atual da região de São Bento do Norte, desde a Ponta dos Três Irmãos até a cidade de Galinhos. Na porção submersa, os arenitos de praia foram observados a cerca de 10 a $20 \mathrm{~km}$ da linha de costa atual, compondo longos trechos retilíneos com algumas dezenas de metros de largura e dezenas de quilômetros de extensão, como mostra a Figura 5.

0 uso das Principais Componentes (PC) foi outro procedimento adotado no processamento das imagens Landsat 7-ETM+. A utilização das PC2, PC4 e PC6 facilitou a identificação da morfologia de fundo e contribuiu para minimizar 0 efeito do sombreamento e dos ruídos existentes. Como 0 uso da Análise por Principais Componentes reduz a dimensionalidade espectral das imagens, para a área submersa observa-se uma concentração das informações referentes a qualidade/profundidade da lâmina d'água na PC4 e PC6, como mostra a elevada participação das bandas 2 e 3 no valor do autovetor dessas PCs (Tab. 1). Já para a PC2, os autovetores apresentados na Tabela 1 demonstram uma ampla diferença na participação das bandas do visível (bandas 1, 2 e 3) em relação àquelas do infravermelho (bandas 4, 5 e 7), o que resulta em elevado contraste entre as feições na zona costeira e na plataforma continental. Quando combinadas em transformação HSI foi possível realçar detalhes de algumas das feições já identificadas, como por exemplo, na composição HSI-PC6-4-2 (Fig. 5). Todas as seqüências de arenitos de praia 


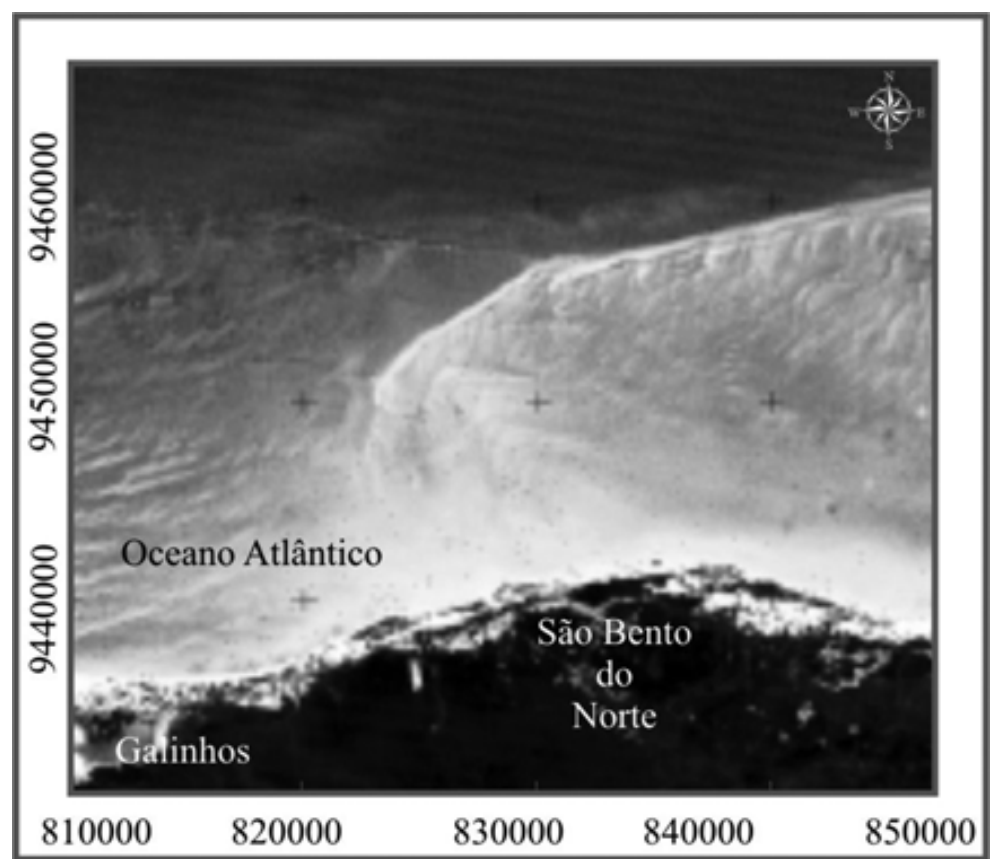

Figura 6 - PC2 da cena Landsat 7-ETM+ (WRS 215/064, adquirida em 12 de junho de 2000, baixamar 7:41Hrs/AM, passagem do satélite aproximadamente 10:00 Hrs/AM $\pm 15 \mathrm{~min}$ ) mostrando plumas em suspensão próxima à linha de costa, linhas de arenitos de praia, dunas e corpo arenoso.

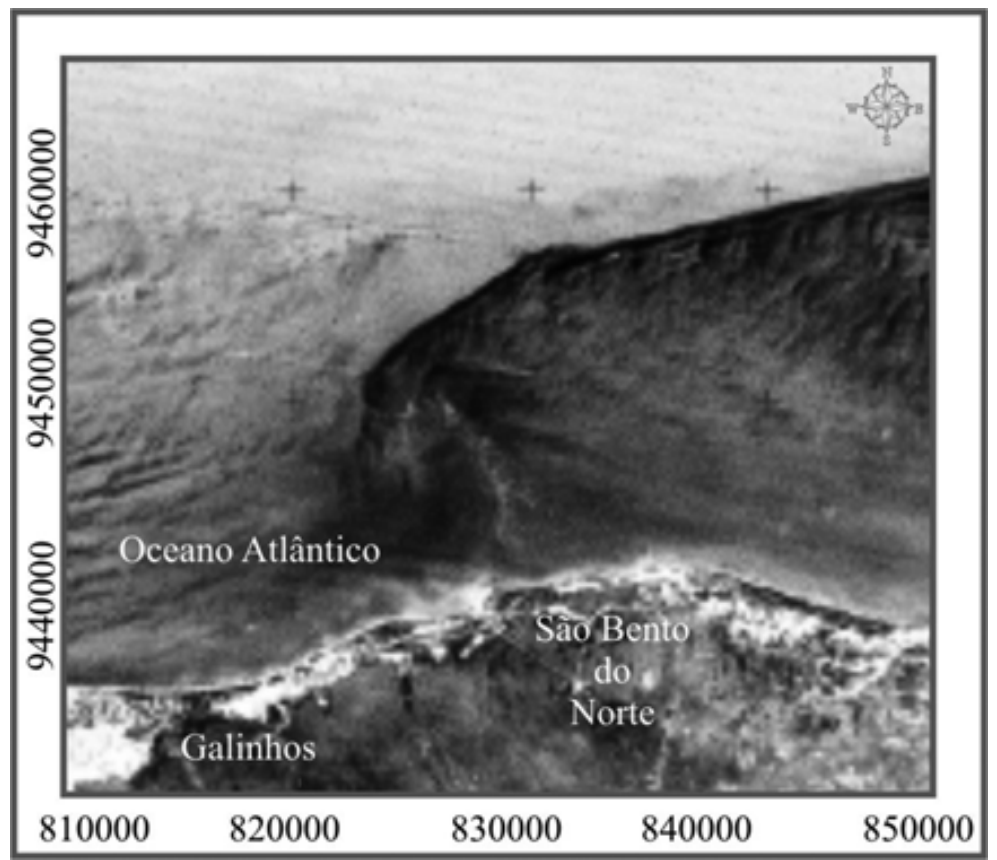

Figura 7 - PC4 da cena Landsat 7-ETM+ (WRS 215/064, de 12 de Junho de 2000, baixamar 7:41Hrs/AM. Passagem do satélite aproximadamente às 10:00 $\mathrm{Hrs} / \mathrm{AM} \pm 15 \mathrm{~min})$, nesta PC foi possível caracterizar com mais detalhes alguns aspectos da porção submersa como: 0 alto topográfico, linhas de dunas paralelas na porção interna do alto topográfico, a continuidade do conjunto de dunas a W, plumas de sedimentos em suspensão ao longo da costa e um maior contraste entre a porção emersa e submersa. 
(ponto 1) e suas variáveis (área de influência, sinuosidade das cristas, orientação e dimensão dos corpos), posicionamento dos recifes (ponto 2), as dunas (alinhamento e comprimento; ponto 3 e 4), uma extensa faixa túrbida de sedimentos em suspensão (ponto 5), um grande corpo arenoso subaquoso (ponto 6) contato entre um substrato marinho de mais alta profundidade com uma zona de baixa profundidade orientado aproximadamente NNW (ponto 7) e que teve sua continuidade continental recoberta por sedimentos recentes (dunas móveis, Fig. 3, Qd; e Fig. 4, ponto 1) e 0 corpo arenoso (Fig. 5, ponto 6), localizado na porção centosuperior da imagem, são melhor realçados.

Durante o desenvolvimento deste estudo outras combinações multiespectrais foram elaboradas. Entretanto, as respostas obtidas nestas combinações (RGB-3-2-1 e RGB-5/2-4-1) e nas imagens monocanais apresentaram informações que confirmam aquelas descritas anteriormente.

\section{CONCLUSÕES}

Baseado na combinação colorida de canais de imagens espectrais do Landsat 7-ETM+ (Órbita 215/064, de 12/Junho/2005), foi possível caracterizar algumas das feições envolvidas na evolução da dinâmica costeira e da geologia local. Estas feições aparecem destacadas no contexto geomorfológico local, no arranjo espacial das feições e na distribuição e dispersão de sedimentos.

Na combinação RGB-5-2-1 o maior contraste foi observado entre a porção emersa e submersa, destacado pela forma da linha de costa, com o ressalto na tonalidade clara das áreas emersas divergindo das áreas recoberta por lâmina d'água que aparecem em tonalidade escura (cinza a preto); na porção continental identificaram-se agrupamentos de extratos vegetais, caracterizados por vegetação de dunas e de áreas cultivadas, campos de dunas e pequenos lagos interdunares.

Na composição HSI-PC6-4-2 e nas PC2 e PC4 destacaramse feições contidas na plataforma continental, onde arenitos de praia (recifes), corpo arenoso, dunas e zonas túrbidas foram diferenciadas com grande nitidez e exatidão.

A partir de uma avaliação regional, pode-se dizer que a plataforma interna adjacente à região de São Bento do Norte está compartimentada em diversos domínios morfológicos. Uma das principais feições identificadas na plataforma é à presença de grandes dunas posicionadas a cerca de $10 \mathrm{~km}$ a norte da costa de São Bento do Norte; estas dunas normalmente estão dispostas de forma assimétrica e orientadas obliquamente (cerca de $10^{\circ}$ ) com relação à linha de costa atual.

Na porção oeste da área (próximo ao corpo arenoso), destaca- se a presença de dunas com dimensões e orientação variadas; estas dunas podem apresentar comprimento na ordem de grandeza desde algumas dezenas de metros até centena de metros. Em geral, estas dunas apresentam orientação N-NE, condizente com o fluxo de massas d'água produzido pela deriva litorânea que na região é de leste para oeste. Também foi possível mapear dunas de menor porte com orientação principal N-S. Em alguns pontos da área de pesquisa foi observado que estas dunas podem ocorrer superpondo as de maior dimensão.

Linhas de arenitos de praia (recifes) alinhados paralelamente à linha de costa atual também podem ser mapeados na área. Estes recifes são encontrados em diversos pontos da plataforma interna estudada, deste a zona de surfe até a quebra da plataforma. Em geral, os registros sonográficos, batimétricos e imagens Landsat 7-ETM+ (Vital et al., 2002; Tabosa \& Vital, 2004; Tabosa et al., 2005; Santos et al., 2007) indicam a presença de corpos levemente alongados e descontínuos, constituídos por algumas dezenas de quilômetros de comprimento.

Os resultados demonstram a importância da utilização de produtos de sensoriamento remoto orbitais, submetidas a tratamento digital, na caracterização de feições submersas e zonas costeiras com características similares àquelas do Litoral Setentrional do RN.

A baixa profundidade da lâmina d'água e a sua transparência contribuíram para o reconhecimento de feições do fundo marinho que até então só eram possíveis com auxilio de técnicas de mergulho, aplicação de métodos acústicos e sísmica rasa. Com o emprego das técnicas de SR/PDI, tem se buscado ampliar as áreas de investigação para níveis de escala regional e de detaIhe, com razões de investimento inversamente proporcionais ao grau de detalhamentos dos trabalhos, o tempo empregado nas investigações e na logística desenvolvida.

Em síntese, pode-se afirma que a composição RGB-5-2-1 foi a que melhor evidenciou os diferentes elementos da paisagem contidos na porção continental da área, entretanto, as formas de fundo presentes na zona marinha, foram destacas quando utilizadas composições do tipo HSI-PC6-4-2 e nas imagens monocanais de PC2 e PC4.

\section{AGRADECIMENTOS}

Os autores agradecem às Instituições: UFRN - Universidade Federal do Rio Grande do Norte, PPGG - Programa de Pósgraduação em Geodinâmica e Geofísica pela infra-estrutura; a ANP - Agência Nacional do Petróleo (PRH-22) pela concessão de bolsa de mestrado ao primeiro autor e aos agentes Finan- 
ciadores: Agência Nacional do Petróleo - ANP; Financiadora de Estudos e Projetos - FINEP, através do Programa de Recursos Humanos da ANP para 0 setor Petróleo e Gás Natural PRH-ANP/MME/MCT, aos projetos MAR-RN (FINEP-CTINFRA) e Projeto Cooperativo PETRORISCO (Rede Cooperativa NorteNordeste de Monitoramento Ambiental, REDE 05 - PETROMAR (FINEP-CTPETRO/PETROBRAS/ CNPq); convênio de cooperação entre Brasil e Alemanha, envolvendo a UFRN e a Christian Albrechts Universität zu Kiel (PROBRAL CAPES/DAAD 150-02), e "Küstenentwicklung und Heutige Küstendynamik in Rio Grande do Norte-NE-Brasilien" (GTZ/DFG).

Apoio Financeiro: MAR-RN (FINEP-CTINFRA), PROBRAL 150-02 (CAPES/DAAD), GTZ/DFG, Agência Nacional do Petróleo - PRH22, Projeto Cooperativo PETRORISCO (Rede Cooperativa Norte-Nordeste de Monitoramento Ambiental, REDE05PETROMAR, FINEP-CTPETRO/PETROBRAS/CNPq).

\section{REFERÊNCIAS}

ALVES AL, AMARO VE \& VITAL H. 2003. Multitemporal analysis of multispectral Landsat 5 - thematic mapper images for monitoring and evaIuation of coastal morphodynamic on the Northeastern Coast of Brazil. Journal of Coastal Research, Brazilian Sandy Beaches, Special Publication, SI 35: 279-283.

ARARIPE PT \& FEIJÓ FJ. 1994. Bacia potiguar. Rio de Janeiro, Boletim de Geociências da PETROBRAS, 8(1): 127-141.

ASSUMPÇÃO M. 1992. The regional intraplate stress field in South America. Journal of Geophysical Research, 97(B8): 11889-11903.

BERTANI RT, COSTA IG \& MATOS RMD. 1990. Evolução tectono-sedimentar, estilo estrutural e habitat do petróleo na bacia potiguar. In: RAJA GABAGLIA GP \& MILANI EJ (Ed.). Origem e Evolução de Bacias Sedimentares. PETROBRAS/SDRH, Rio de Janeiro, 291-310.

BEZERRA FHR, LIMA FILHO FP, AMARAL RF, CALDAS LHO \& COSTA NETO LX. 1998. Holocene coastal tectonics in NE Brazil. In: STEWART I \& VITA-FINZI C (Ed.). Coastal Tectonics Geol. Soc. London, Special Publication, 146: 279-293.

CALDAS LHO, STATTEGGER K \& VITAL H. 2006. Holocene sea-level history and coastal evolution: evidences from coastal sediments of the northern Rio Grande do Norte coast, NE Brazil. Marine Geology, Amsterdam, 228 (1-4): 39-53.

COSTA NETO LX. 1997. Evolução geológica-geomorfológica recente da plataforma continental interna ao largo do delta do Rio Açu, Macau - RN. Programa de pós-graduação em geologia e geofísica marinha da Universidade Federal Fluminense, Rio de Janeiro, Dissertação de Mestrado, $214 \mathrm{pp}$.
CREMONI OA. 1995. A reativação Tectônica da Bacia Potiguar no Cretáceo Superior. SBG, Boletim de resumos expandido, V Simpósio Nacional de Estudos Tectônicos, 1: 277-280, Gramado, RS.

DALRYMPLE RW \& RHODES RN. 1995. Estuarine dunes and barforms. In: PERILLO GM (Ed.). Geomorphology and Sedimentology of Estuaries. Developments in Sedimentology, Elsevier, 53: 359-422.

FERREIRA JM, OLIVEIRA RT, TAKEYA MK \& ASSUMPÇÃO M. 1998. Superposition of local and region stresses in Northeast Brazil: Evidence from focal mechanisms around the potiguar marginal basin. Geophysics Journal International, 134: 341-355.

FONSECA VP. 1996. Estudo morfo-neotectônico na área do baixo curso do Rio Açu (Assu-Macau), Rio Grande do Norte. IG/UFMG, Belo Horizonte, Dissertação de mestrado, 109 pp.

GUYOT G. 1989. Signatures spectrales des surfaces naturelles. Caen, Paradigme. Télédétection Satellitaire, 5: 178 pp.

LIMA ZMC, ALVES AL, AMARO VE \& VITAL H. 2001. Evolução da linha de costa do esporão de Galinhos (NE Brasil) utilizando fotografias aéreas e imagens Landsat TM. Revista Pesquisas em Geociências, 28 : 497-507.

LIMA ZMC. 2004. Caracterização da dinâmica ambiental da região costeira do município de Galinhos, litoral setentrional do Rio Grande do Norte. Programa de Pós-Graduação em Geodinâmica e Geofísica da Universidade Federal do Rio Grande do Norte, Natal, RN. Tese de Doutoramento, $144 \mathrm{pp}$.

MATOS RMD. 1987. Sistema de rífts cretáceos do NE brasileiro. In: Seminário de Tectônica da Petrobras (TECTOS I). Rio de Janeiro. Atas... Rio de Janeiro, PETROBRAS/DEPEX, 125-159.

MATOS RMD. 1992. The northeast brazilian rift system. Tectonics, 11(4): 766-791.

OLIVEIRA DC, CRUZ Jr FW \& MENEZES RMRF. 1996. Significado geodinâmico da estruturação rasa no extremo sudoeste da Bacia Potiguar (Nordeste do Brasil). Boletim de resumos, IV Simpósio sobre o Cretáceo do Brasil, 1: 95-106, UNESP, Rio Claro, SP.

RÖBER V. 2001. Structure and dynamics of the inner shelf north of Galinhos, Rio Grande do Norte (NE - Brazil). Institute of Geosciences, University Christian Albrechts zu Kiel, Germany. Tese, 76 pp.

SANTOS CLA, VITAL H, AMARO VE \& KIKUCHY RKP de. 2007. Mapeamento de recifes submersos na costa do Rio Grande do Norte, NE Brasil: Macau a Maracajau. Revista Brasileira de Geofísica. 25 (Supl. 1): 27-36.

SCHWARZER K, STATTEGGER K, VITAL H \& BECKER M. 2006. Holocene coastal evolution of the Rio Açu area (Rio Grande do Norte, Brazil). Journal of Coastal Research, USA, Special Publication, SI 39: 140-144.

SHORT AD. 1999. Handbook of beach and shoreface morphodynamics. British Library Ed. England. 379 pp. 
SNEDDEN JW \& DALRYMPLE RW. 1999. Modern shelf sand ridges: From historical perspective to a unified hydrodynamic and evolutionary model. In: BERGMAN KM \& SNEDDEN JW (Ed.). Isolated Shallow Marine Sand Bodies: Sequence Stratigraphic Analysis and Sedimentologic Interpretation. Society for Sedimentary Geology, SEPM, Special Publication, 64: 13-28.

SOARES UM, ROSSETI EL \& CASSAB RCT. 2003. Bacias sedimentares brasileiras: Bacia Potiguar. Fundação Paleontológica Phoenix, vol. 56. $8 \mathrm{pp}$.

SOLEWICZ R. 1989. Feições fisiográficas submarinas da plataforma continental do Rio Grande do Norte visível por imagens de satélite. Instituto de Pesquisas Espaciais. São José dos Campos, São Paulo, Dissertação de Mestrado, $143 \mathrm{pp}$.

SOUZA SM. 1982. Análise litoestratigráfica da bacia potiguar. In: SBG, Congresso Brasileiro de Geologia, 32, Salvador, anais, 5: 2.392-2.406.

STATTEGGER K, CALDAS LHO \& VITAL H. 2006. Holocene coastal evolution of the Northern Rio Grande do Norte Coast, Brazil. Journal of Coastal Research, Proceedings of the 8th International coastal symposium, USA, Special Publication, SI 39: 150-155.

TABOSA WF. 2000. Dinâmica costeira da região de São Bento do Norte e Caiçara do Norte - RN. Departamento de Geologia, Universidade Federal do Rio Grande do Norte, Relatório de Graduação, 76 pp.

TABOSA WF \& VITAL H. 2004. Interpretações preliminares para dados hidrodinâmicos da plataforma interna adjacente a São Bento do Norte RN. In: Congresso Brasileiro de Oceanografia. Itajaí, SC, p. 436.

TABOSA WF, LIMA ZMC, VITAL H \& GUEDES IMG. 2001a. Monitoramento costeiro das praias de São Bento do Norte e Caiçara do Norte NE Brasil. Revista Pesquisas em Geociências, 28: 383-392.

TABOSA WF, CALDAS LHO, VITAL H \& AMARO VE. 2001b. Remote sensing of the northeastern brazilian continental margin (São Bento/Caiçara do Norte). In: The American Geophysical Union. Chapman Conference on the Formation of Sedimentary Strata on Continental Margins. Ponce, Puerto Rico, AGU, 37. $46 \mathrm{pp}$.

TABOSA WF, VITAL H \& AMARO VE. 2002. Morphologic and structural characterization of the Rio Grande do Norte northern coast, Northeast
Brazil on Remote Sensing Images. In: American Association of Petroleum Geologist, Houston, Texas, USA, 1: 51. 198 pp. CD-ROM.

TABOSA WF, VITAL H, AMARO VE \& SANTOS NETO F. 2005. Aplicação de métodos acústicos na interpretação de zonas submersas no litoral setentrional do RN. Resumo expandido, X Congresso da ABEQUA. Guarapari, ES, Boletim de resumos expandido, CD-ROM.

TAYLOR RM. 1982. Color in soils and sediments. Review. In: OLPHEN H \& VIENALE F (Ed.). Clay Conf., Bologna 1981, Elsevier, Amsterdam. 705-761.

TESTA V \& BOSENCE DWJ. 1998. Carbonate-siliciclastic sedimentation on high-energy, ocean-facing, tropical ramp, NE Brazil. In: WRIGHT VP \& BURCHETTE TP (Ed.). Carbonate Ramps. Geol. Soc. London Special Publication, 149: 55-71.

TESTA V \& BOSENCE DWJ. 1999. Physical and biological controls on the formation of carbonate and siliciclastic bedforms on the North-East Brazilian: Shelf. Sedimentology, 46: 279-301.

VIANNA ML, SOLEWICZ R, CABRAL A \& TESTA V. 1991. Sandstream on the Northeast Brazilian Shelf. Continental Shelf Research, 2: 509-524.

VITAL H, AMARO VE, TABOSA WF, GUEDES IMG, STATTEGGER K \& CALDAS LHO 2002. Pattern of sediment distribution in tectonics setentrional coast of Rio Grande do Norte state, Northeastern Brazil. Published as a supplement to Eos, Transactions, American Geophysical Union. 2002 Ocean Sciences Meeting. Vol. 83, AGU, 4: OS17; Honolulu, Hawaii, USA.

VITAL H, STATTEGGER K, TABOSA WF \& RIEDEL K. 2003. Why does erosion occur on the northeastern coast of Brazil?: The Caiçara do Norte Beach example. Journal of Coastal Research, Special Publication, SI 35: 525-529, Itajaí, SC.

VITAL H. 2005. Rio Grande do Norte. In: MUEHE D (Ed.). Erosão e Progradação do Litoral Brasileiro. MMA. P. 159-176.

VITAL H, AMARO VE \& SILVEIRA IM. 2006. Coastal erosion on the Rio Grande do Norte State (Northeastern Brazil): Causes and Factors Versus Effects and Associated Processes. Journal of Coastal Research, USA, Special Publication, SI 39: 1307-1310. 


\section{NOTAS SOBRE OS AUTORES}

Werner Farkatt Tabosa. Bacharel em Geologia pela Universidade Federal do Rio Grande do Norte - UFRN e Mestre em Dinâmica Costeira pelo Programa de PósGraduação em Geodinâmica e Geofísica - UFRN. Participou durante um ano de um intercâmbio de pesquisa pelo doutorado sanduíche na Christian Albrechts Universität zu Kiel, Alemanha e neste momento está concluindo sua tese de doutorado pelo Programa de Pós-Graduação em Geodinâmica e Geofísica - UFRN. Bolsista da Agência Nacional do Petróleo Dsc.2: ANP-MME-PRH22.

Venerando Eustáquio Amaro. Bacharel em Geologia pela Universidade Federal do Mato Grosso e Mestre em Geologia Econômica pela Universidade Federal de Brasília - UNB. Participou durante um ano de um intercâmbio de pesquisa pelo doutorado sanduíche na Université Claude Bernard - Lyon I, França e concluiu sua tese de doutorado em Sensoriamento Remoto em 1998 na Universidade de São Paulo e tem trabalhado com Geoprocessamento na Universidade Federal do Rio Grande do Norte desde 1989.

Helenice Vital. Bacharel em Geologia pela Universidade Federal do Rio Grande do Norte e Mestre em Geologia Marinha e Ambiental pela Universidade Federal do Pará - UFPA. Concluiu sua tese de doutorado em 1996 na Christian Albrechts Universität zu Kiel, Alemanha e tem trabalhado com Geologia e Geofísica Marinha na Universidade Federal do Rio Grande do Norte. Sendo Pesquisadora do CNPq desde 1999. 